\title{
Carbon nanotube network formation from evaporating sessile drops
}

William R. Small ${ }^{1 *}$, Chris D. Walton ${ }^{1}$, Joachim Loos ${ }^{2}$, and Marc in het Panhuis ${ }^{1 *}$

1. Department of Physical Sciences, the University of Hull, Hull, HU6 7RX, United Kingdom

2. Department of Chemical Engineering and Chemistry, Eindhoven University of Technology, P.O. Box 513, 5600 MB Eindhoven, The Netherlands

* Authors to whom correspondence should be addressed. E-mail: W.Small@chem.hull.ac.uk, M.Panhuis@hull.ac.uk

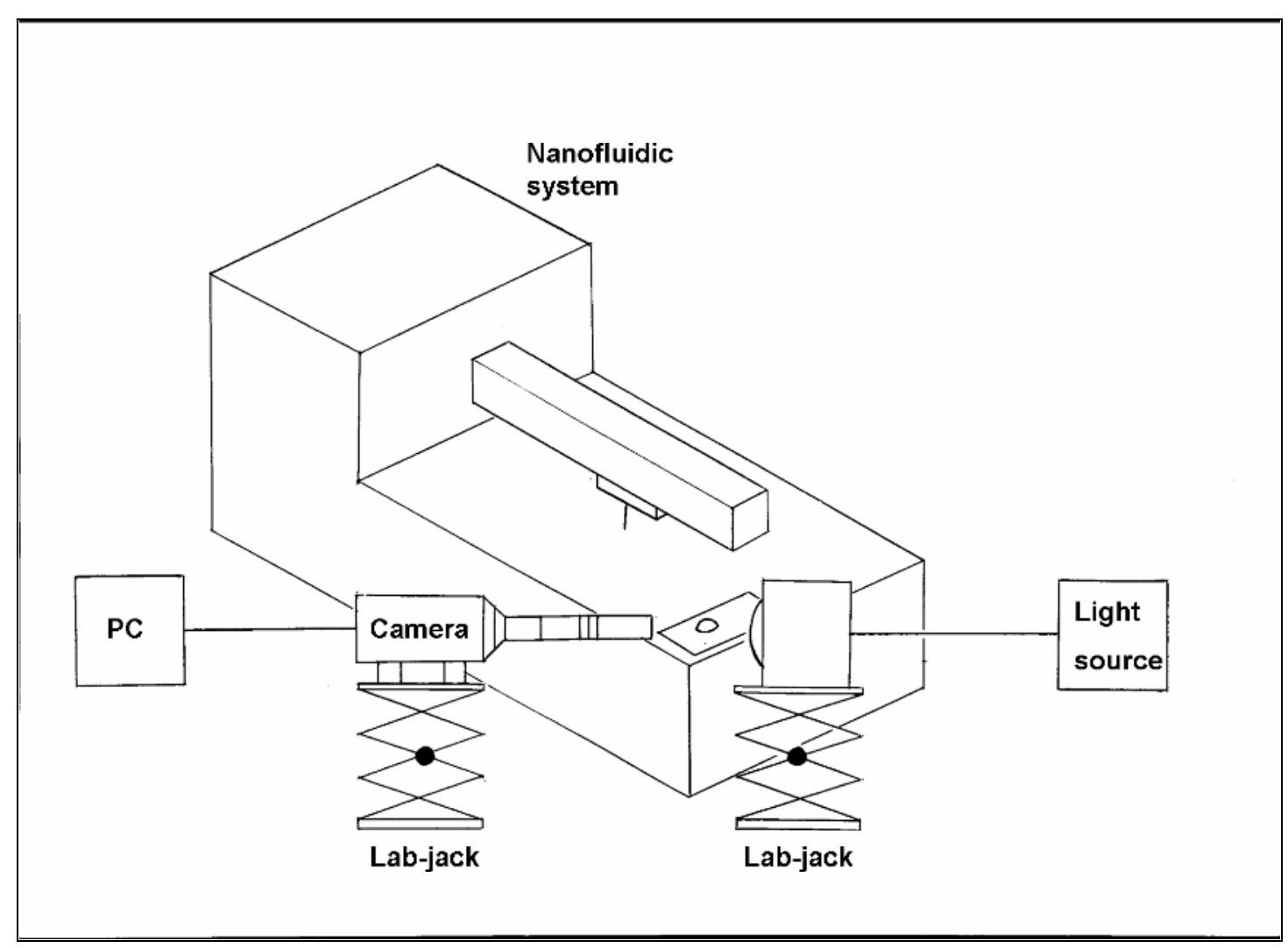

Figure S1: Schematic of instrument set-up for the in situ analysis of contact angles of $100 \mathrm{~nL}$ sessile drops. 


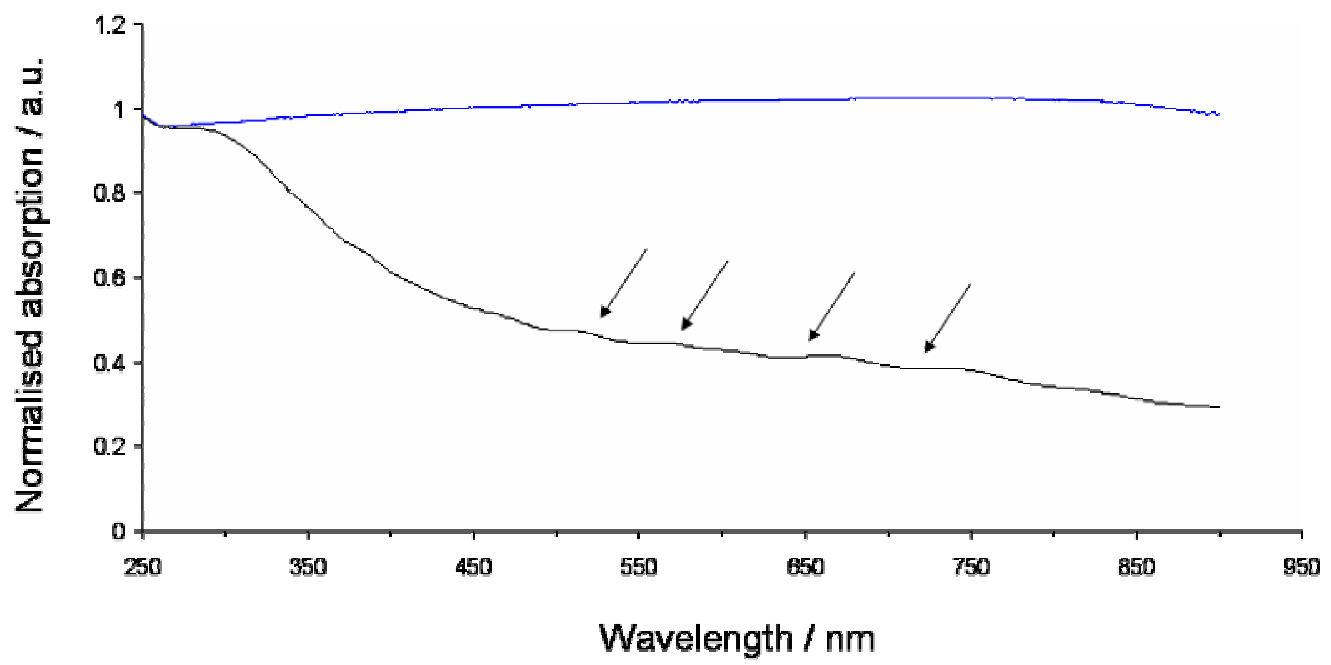

Figure S2: UV-visible spectra (normalized) of $0.25 \%(\mathrm{w} / \mathrm{v})$ SDS-SWNT as-prepared (blue line) and centrifuged (black line) dispersions. Arrows indicate broad absorption features due to nanotubes.
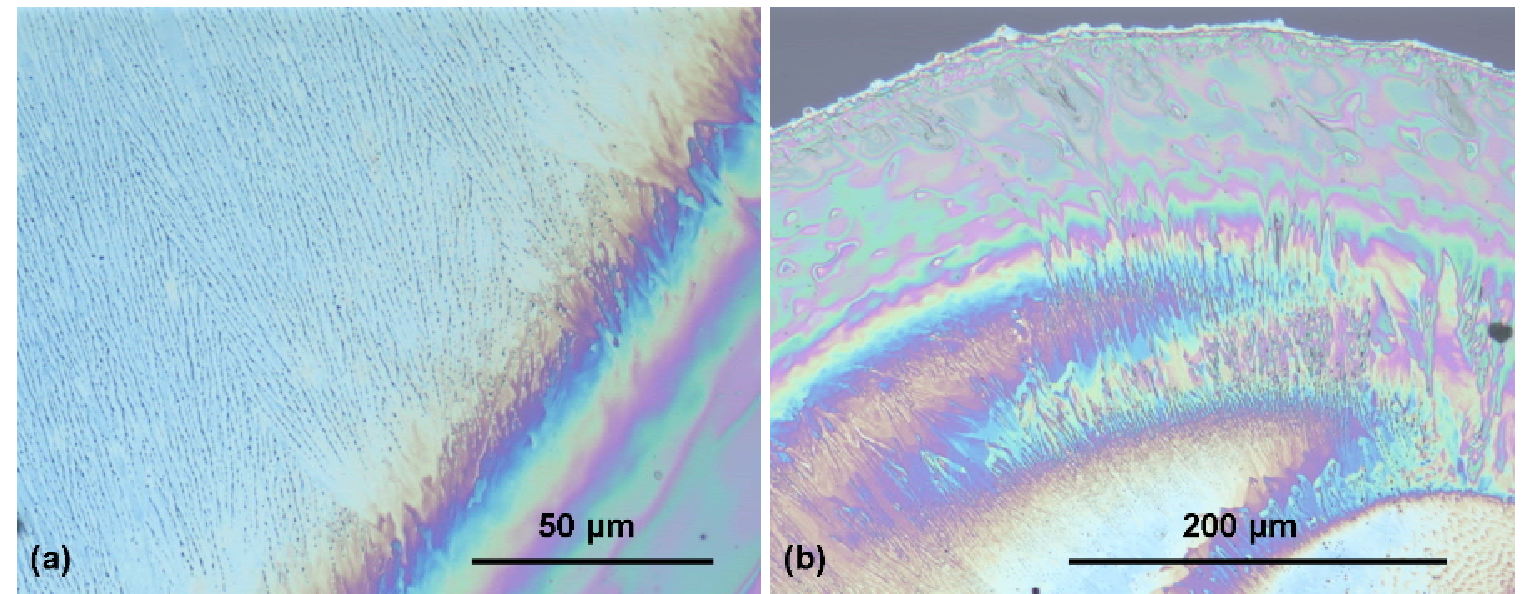

Figure S3: Optical microscopy images of evaporated drop (deposited from $100 \mathrm{nl} 1.0$ $\%(w / v)$ SDS solution) showing linear striations (a) and liquid-crystal type structures (b). 

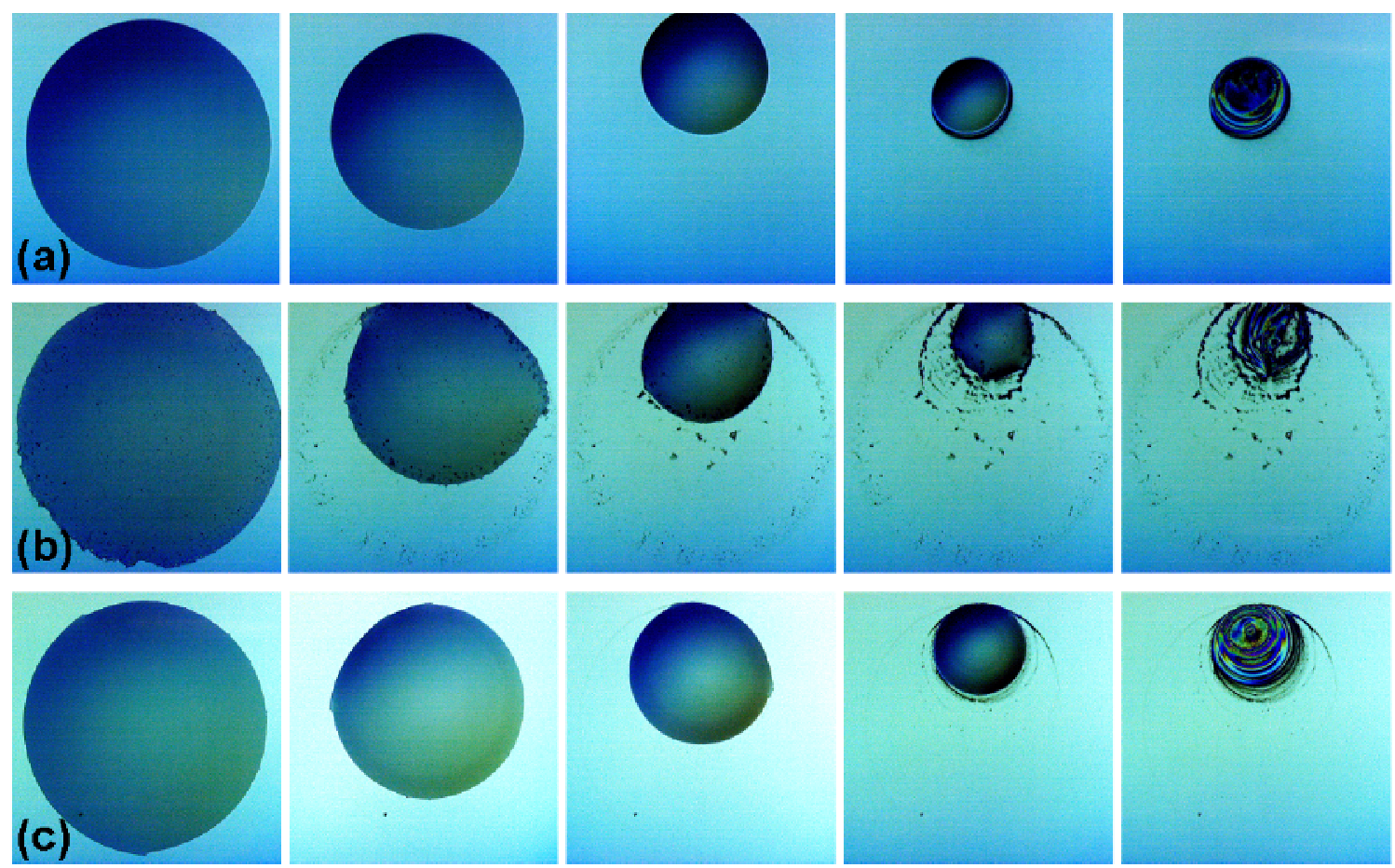

$t=0$

$t=\mathbf{2 5}$

$\mathbf{t}=\mathbf{5 0}$

$t=75$

$t=100$

Figure S4: Video microscopy images of evaporating $100 \mathrm{~nL}$ drops on untreated silicon. (a) SDS solution, (b) as-prepared SDS-SWNT dispersion and (c) centrifuged SDS-SWNT dispersion. Numbers beneath images indicate time in seconds. SDS concentration $0.25 \%(\mathrm{w} / \mathrm{v})$.

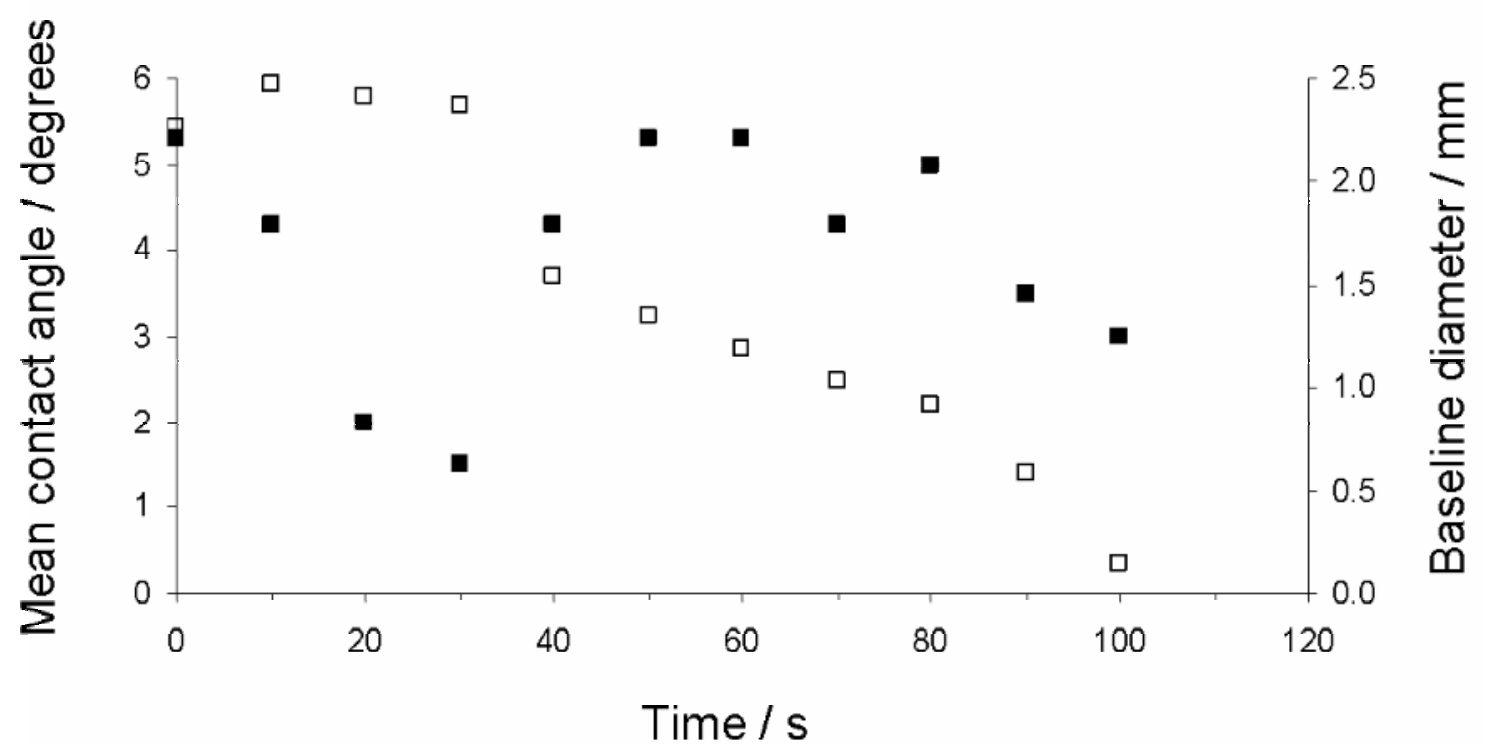


Figure S5: Mean contact angle (filled squares) and baseline diameter (empty squares) of an evaporating $100 \mathrm{~nL}$ drop (deposited from an as-prepared $0.25 \%(\mathrm{w} / \mathrm{v})$ SDSSWNT dispersion) on untreated silicon.

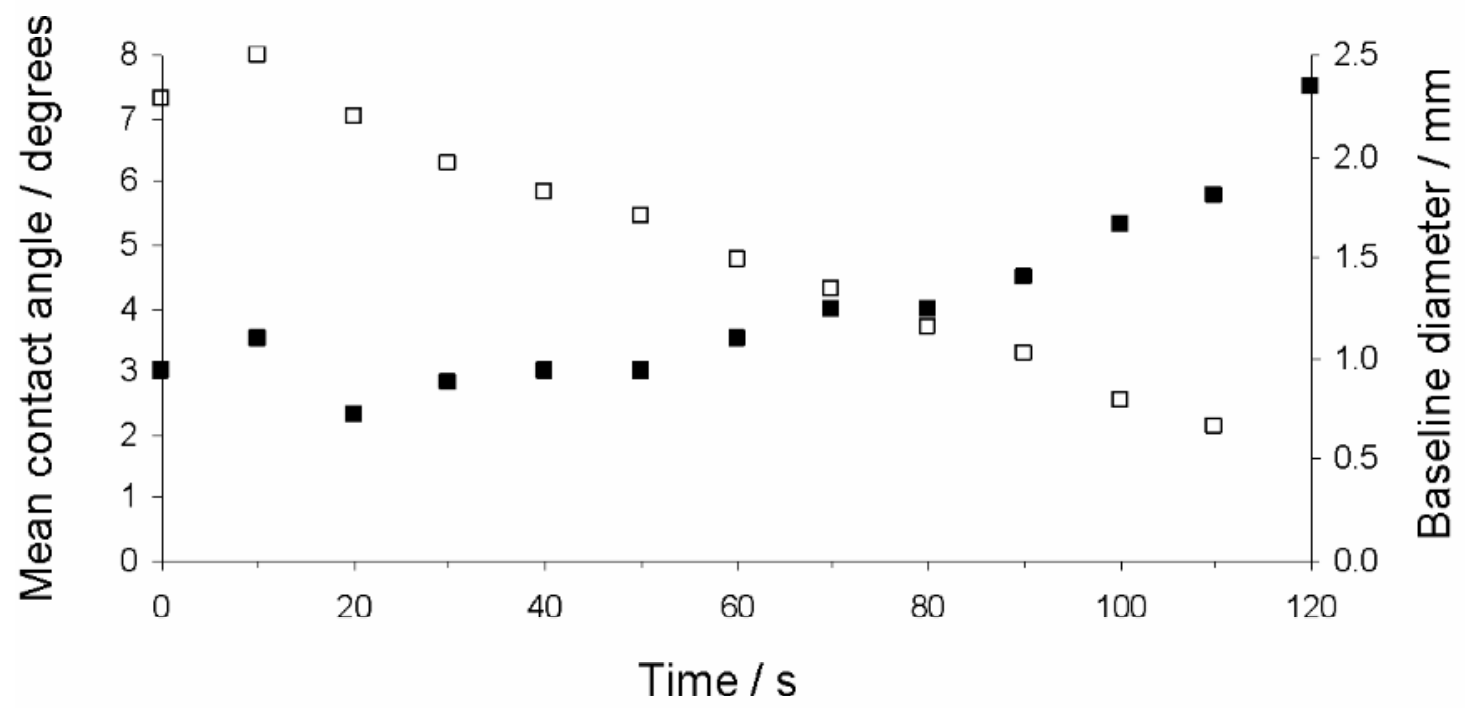

Figure S6: Mean contact angle (filled squares) and baseline diameter (empty squares) of an evaporating $100 \mathrm{~nL}$ drop (deposited from a centrifuged $0.25 \%(\mathrm{w} / \mathrm{v})$ SDSSWNT dispersion) on untreated silicon. 

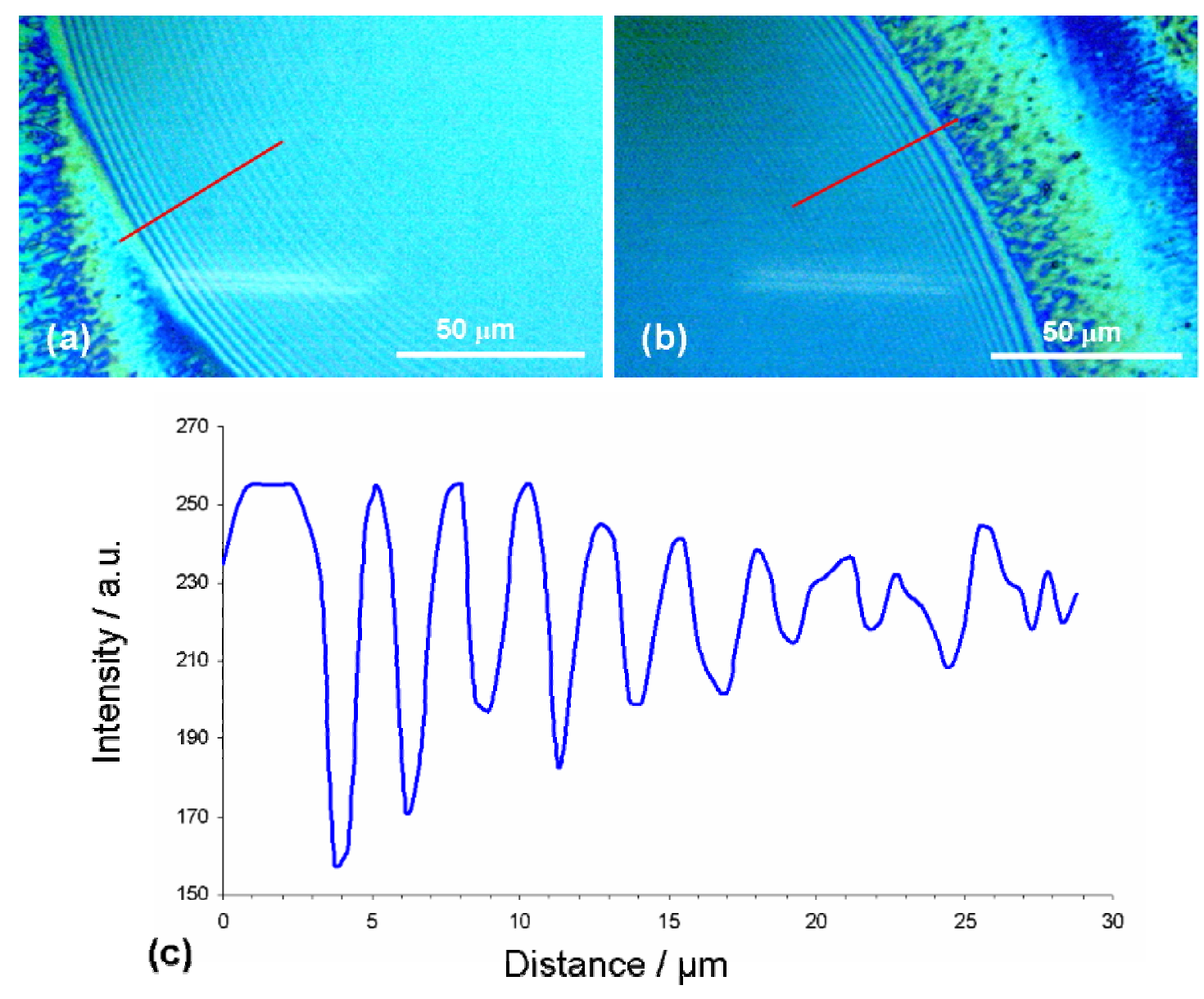

Figure S7: Optical microscopy images of $100 \mathrm{~nL}$ drops (deposited from a centrifuged $0.25 \%(w / v)$ SDS-SWNT dispersion) on (a) untreated and (b) OTS-treated silicon substrates. Red lines show where line-profiles were obtained. (c) Example of a typical line profile, showing light intensity as a function of distance from film edge. 


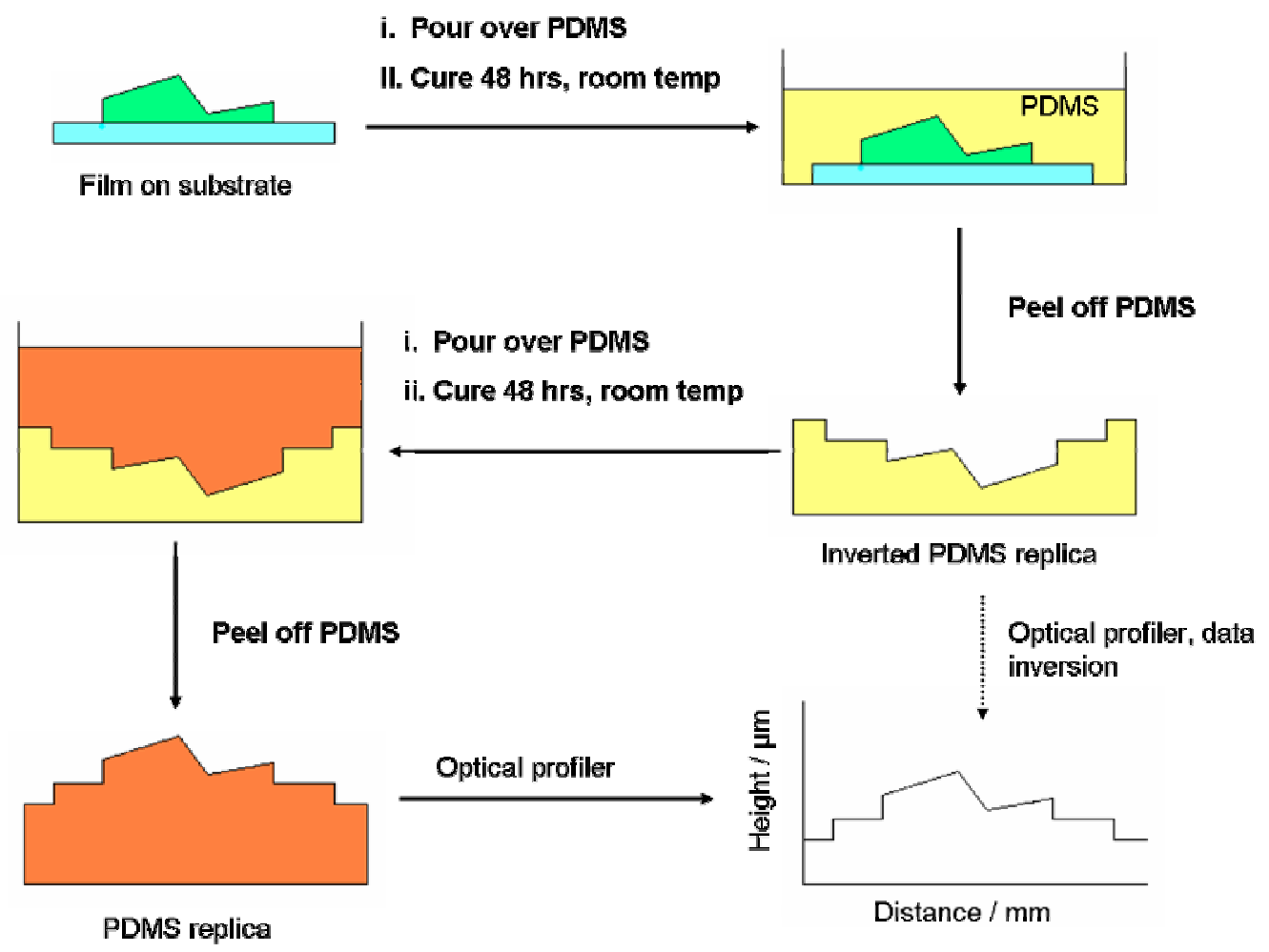

Figure S8: Schematic of the PDMS replication process of evaporated drops. 

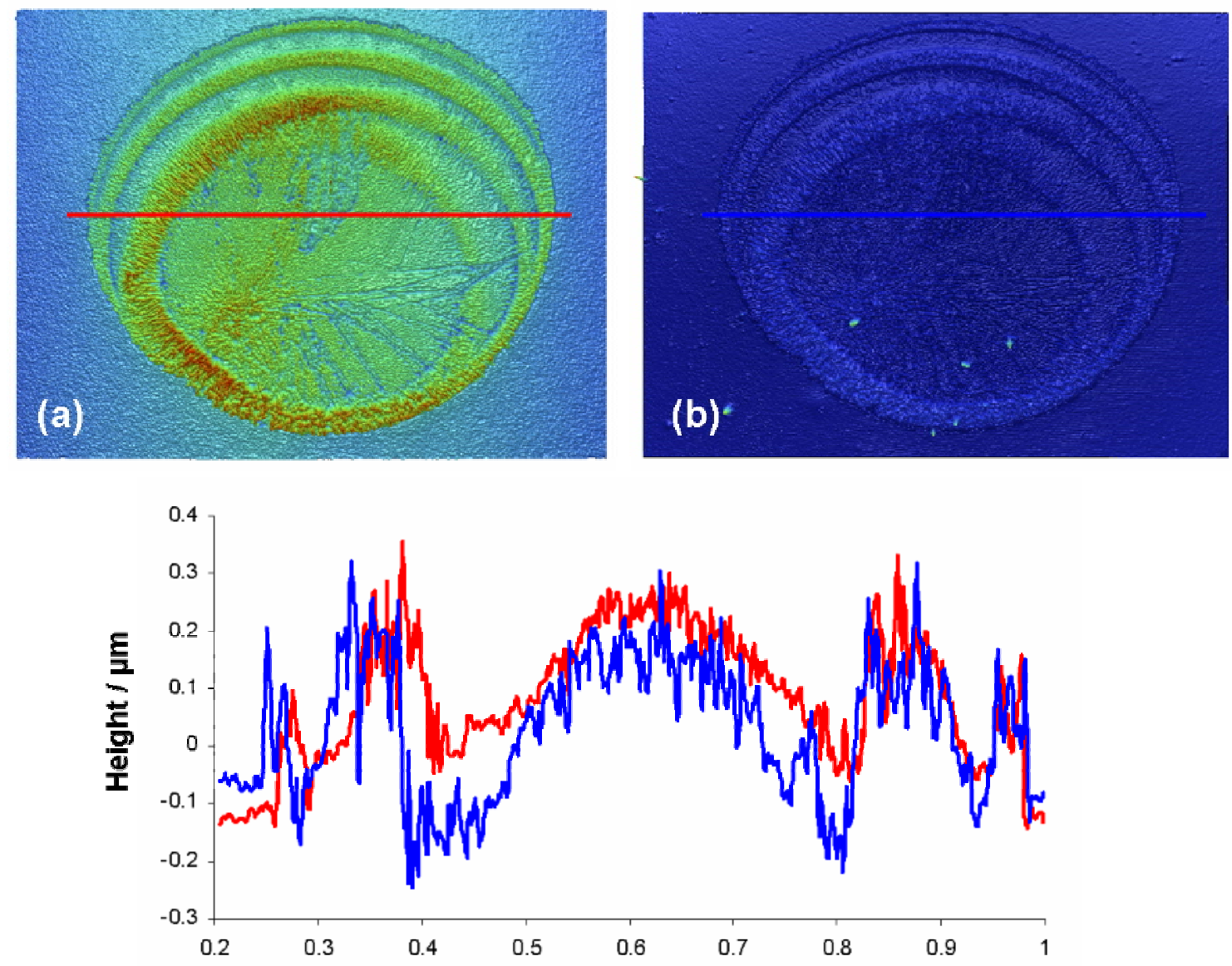

(c)

\section{Distance / $\mathrm{mm}$}

Figure S9: Topographical analysis on PMDS replica of an evaporated drop using a single (a) and a double (b) replication process. (c) Corresponding height profiles of single (red line) and double (blue line) replication processes. 

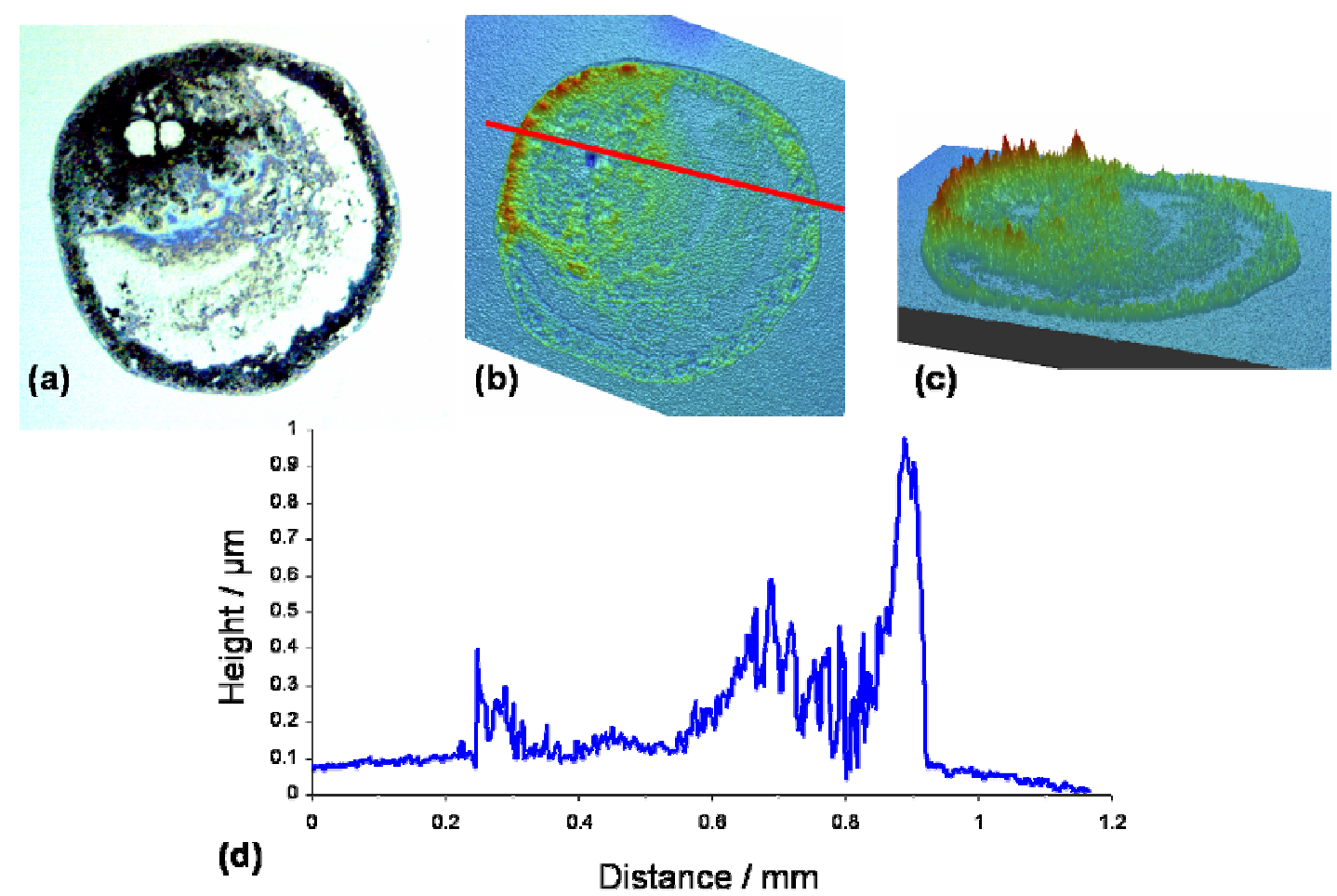

Figure S10: (a) Optical microscopy image of an evaporated drop (deposited from an as-prepared $0.25 \%(\mathrm{w} / \mathrm{v})$ SDS-SWNT dispersion) on OTS-treated silicon. Corresponding topographical analysis on PDMS replica: (b) 2D view in same orientation as optical image, (c) 3D view and (d) height profile (obtained over red line) as a function of drop diameter. 
The amount of surfactant removed can be semi-quantitatively determined by redispersing the sediment in water (normally discarded after centrifugation) and comparing it with freshly prepared SWNT dispersions as a function of SDS concentration. Visual observations suggest that the redispersed sediment is in the range of (freshly prepared) $0.05 \%-0.10 \%$ SDS-SWNT (see Figure S10), suggesting an SDS concentration that is in good agreement with the calculated estimates (see main text).

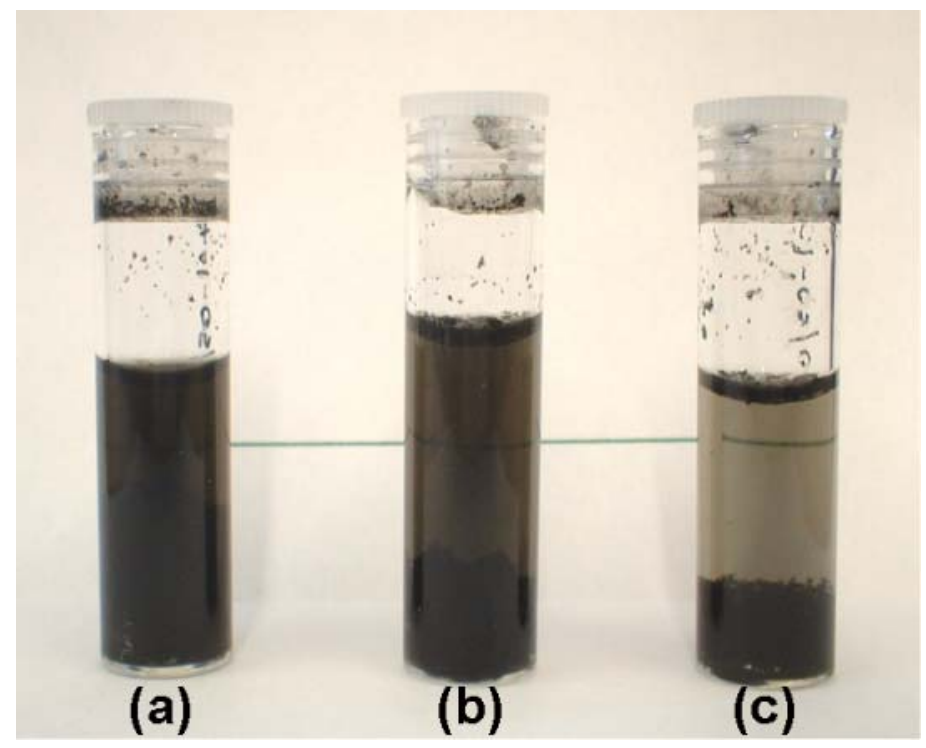

Figure S11: Photographs of (a) freshly prepared 0.10\% SDS-SWNT dispersion (b) redispersed sediment obtained after centrifugation of a $0.25 \%(\mathrm{w} / \mathrm{v})$ SDS-SWNT dispersion and (c) freshly prepared $0.05 \%(\mathrm{w} / \mathrm{v})$ SDS-SWNT dispersion. 